\title{
Dairy consumption and cardiovascular disease events, bone fracture and all-cause mortality
}

Dairy products are important constituents of a healthy and balanced diet, and their association with health outcomes remains to be established ${ }^{(1)}$. To provide further evidence on the associations between different dairy subtypes and health outcomes, we investigated the association of total dairy, different dairy subtypes (total fermented dairy, full-/low-fat milk, yogurt, cheese, butter and cream) and risk of cardiovascular disease (CVD), coronary heart disease (CHD), bone fracture and all-cause mortality among 1746 Danish men and women from the WHO-initiated survey Monitoring of Trends and Determinants in Cardiovascular Diseases (MONICA) cohort.

Dairy consumption was collected by using 7-day weighted diet diaries ${ }^{(2)}$. The incidence of outcome events was defined according to the International Classification of Diseases and were identified by recorded linkage to the National Patient Registry ${ }^{(3)}$. Hazard ratios (HRs) and 95\% CIs were estimated using the multivariable Cox proportional hazard models.

During a mean follow-up of 30 years, incident case of CVD $(n=904)$, CHD $(n=332)$, fracture $(n=447)$ and all-cause mortality $(n$ $=680)$ were reported. High intake of total fermented dairy was associated with lower fracture risk $(\mathrm{HR} 0.67,95 \% \mathrm{CI}: 0.51-0.90, P=$ 0.02) than observed in the lowest tertile of the fermented dairy group. Furthermore, high intake of low-fat milk was associated with lower risks of CVD (HR 0.84, 95\% CI: 0.68-1.03, $P=0.03$ ), CHD (HR 0.82, 95\% CI: 0.59-1.16, $P=0.04$ ), and all-cause mortality (HR $0.77,95 \%$ CI: $0.61-0.97, P=0.004$ ) compared with the lowest tertile of low-fat milk group. No associations were found with other dairy subtypes.

The findings from this prospective cohort study suggest an inverse association between total fermented dairy and fracture risk, and also inverse associations were found between low-fat milk consumption and risk of CVD, CHD and all-cause mortality. However, the mechanisms linking fermented dairy and low-fat milk consumption to the health outcomes are not clear. Healthy lifestyle or the specific foods dairy products replace in the general diet may contribute to the detected associations and this needs future research to confirm.

\section{References}

1. Soedamah-Muthu SS, Guo J, Givens DI, et al. (2020) Milk and dairy foods: their functionality in human health and disease. London: Elsevier Inc. 2. Jakobsen MU, Overvad K, Dyerberg J, et al. (2004) Am J Epidemiol 160, 141-9.

3. Juel K \& K Helweg-Larse (1999) Dan Med Bull 46, 354-7. 